\title{
Fundamental cavity impedance and longitudinal coupled-bunch instabilities at the High Luminosity Large Hadron Collider
}

\author{
P. Baudrenghien ${ }^{1}$ and T. Mastoridis ${ }^{2}$ \\ ${ }^{1}$ CERN, Geneva 1211, Switzerland \\ ${ }^{2}$ California Polytechnic State University, San Luis Obispo, California 93407, USA
}

(Received 3 April 2016; published 19 January 2017)

\begin{abstract}
The interaction between beam dynamics and the radio frequency (rf) station in circular colliders is complex and can lead to longitudinal coupled-bunch instabilities at high beam currents. The excitation of the cavity higher order modes is traditionally damped using passive devices. But the wakefield developed at the cavity fundamental frequency falls in the frequency range of the rf power system and can, in theory, be compensated by modulating the generator drive. Such a regulation is the responsibility of the low-level rf (llrf) system that measures the cavity field (or beam current) and generates the rf power drive. The Large Hadron Collider (LHC) rf was designed for the nominal LHC parameter of 0.55 A DC beam current. At $7 \mathrm{TeV}$ the synchrotron radiation damping time is 13 hours. Damping of the instability growth rates due to the cavity fundamental ( $400.789 \mathrm{MHz}$ ) can only come from the synchrotron tune spread (Landau damping) and will be very small (time constant in the order of $0.1 \mathrm{~s}$ ). In this work, the ability of the present llrf compensation to prevent coupled-bunch instabilities with the planned high luminosity LHC (HiLumi LHC) doubling of the beam current to 1.1 A DC is investigated. The paper conclusions are based on the measured performances of the present llrf system. Models of the rf and llrf systems were developed at the LHC startup. Following comparisons with measurements, the system was parametrized using these models. The parametric model then provides a more realistic estimation of the instability growth rates than an ideal model of the rf blocks. With this modeling approach, the key rf settings can be varied around their set value allowing for a sensitivity analysis (growth rate sensitivity to rf and llrf parameters). Finally, preliminary measurements from the LHC at $0.44 \mathrm{~A} \mathrm{DC}$ are presented to support the conclusions of this work.
\end{abstract}

DOI: 10.1103/PhysRevAccelBeams.20.011004

\section{INTRODUCTION}

The high currents employed in modern light sources and circular accelerators lead to strong coupling of the bunch motion with the cavity impedance, which can overcome Landau and/or synchrotron radiation damping and cause coherent instabilities. The cavity fundamental impedance is of particular concern, since it has to couple strongly to the beam, and therefore leads to a strong beam excitation. Since the beam induced field falls in the bandwidth of the rf power system though, it can be compensated using a modulation of the generator drive. This is the responsibility of the llrf. This work studies instabilities driven by the cavity fundamental impedance.

During the design of the nominal LHC it was concluded that Landau damping would be sufficient to preserve stability in the single rf LHC system [1]. A longitudinal damper was not part of the plan. It should be noted that this study was concerned with broadband longitudinal impedance only $[\operatorname{Im}(Z / n)$ around $0.14 \Omega]$. It was assumed that

Published by the American Physical Society under the terms of the Creative Commons Attribution 3.0 License. Further distribution of this work must maintain attribution to the author $(s)$ and the published article's title, journal citation, and DOI. the fundamental cavity impedance issue could be taken care of by the llrf. It is important to check that this is also correct for the HiLumi LHC.

The interaction between the beam and the rf system is complex and can change significantly over the operating range of accelerator parameters (beam current and energy) and $\mathrm{rf}$ parameters (change of loaded quality factor $Q_{L}$ [2], cavity detuning, generator saturation). A closed form solution for the optimal $\mathrm{rf}$ and llrf parameters is not possible. Therefore, a model approach is preferred to study the beam dynamics interaction with the rf system, estimate stability margins, and evaluate the sensitivity to system parameters. Such an approach has been used before at PEP-II. Coupled-bunch instabilities induced by the fundamental rf system were the performance limiting effects for PEP-II. Using the rf models, the sensitivity of beam instabilities to individual llrf parameters, the effectiveness of alternative operational algorithms, and the possible tradeoffs between rf loop and beam stability were studied [3-5].

Similar studies have been conducted for the nominal LHC current showing a large stability margin [6]. The current in the HiLumi LHC will be higher though, resulting in a twice larger beam loading factor (up to 7.2 at $7 \mathrm{TeV}$ for HiLumi LHC) [7]. Therefore, models of the rf and llrf systems in the HiLumi LHC have been developed, which 
together with the LHC operational experience on $\mathrm{rf} / \mathrm{llrf}$ settings and constraints, allow the estimation of the effective impedance presented to the beam by the rf station for various beam parameters. The coupled-bunch instability threshold can then be computed to study the longitudinal stability due to multibunch coupling as a function of the rf configurations. Estimates of longitudinal coupled-bunch instabilities for the HiLumi LHC are presented [9]. Machine development (MD) studies to quantify the coupled-bunch motion stability margins were also conducted and the results are shown.

Section II describes the llrf loops, whereas Sec. III establishes the formalism for estimating the coupled-bunch instabilities growth rates. Section IV presents the stability criterion. The configurations of interest are described in Sec. V, whereas Section VI includes the growth rates estimated for the HiLumi LHC configurations of interest. The sensitivity of the instability growth rates to rf and llrf parameters is shown in Section VII. Section VIII briefly presents the current threshold due to cavity fundamental driven instabilities. The reduction of stability margin with reduced bunch length is presented in Sec. IX. Finally, Sec. X includes preliminary LHC measurements of instability growth rates used to validate the formalism presented in this work.

\section{LLRF LOOPS}

Two requirements were of particular importance for the design of the LHC low level rf: keeping the rf noise at a very low level and minimizing the effective cavity impedance at the fundamental. This last requirement is very relevant to the present discussion.

Beam loading - the perturbation of the cavity field at the fundamental by the beam-induced voltage- has been a concern in all high-intensity machines, since the late 1970s. The classic solution is to correct for the beam-induced perturbation by modulating the generator current. This can be done by measuring the cavity field using an antenna, by processing the signal via a fast feedback loop, and correcting via a modulation of the generator current [10].

Figure 1 shows the principle of a rf feedback around a cavity. The cavity voltage is compared to the desired voltage. The error is amplified by the low level rf and generates a drive to the generator. The loop delay is represented on the simplified diagram as it is a limitation

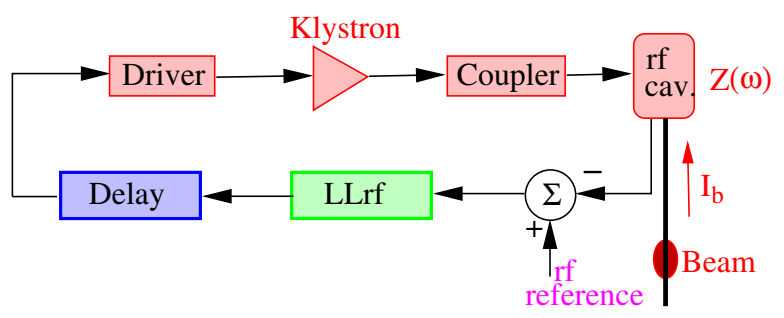

FIG. 1. Rf loop model block diagram. in any feedback system. The LHC has one generator (klystron) per cavity. The rf main coupler represents a transformation of the generator current to the accelerating gap where it adds to the beam current $I_{b}$, to create the cavity voltage via the cavity impedance $Z(\omega)$.

$Z_{\mathrm{cl}}(\omega)$ is defined as the close-loop cavity impedance, which is the cavity impedance seen by the beam in the presence of feedback. From Controls theory:

$$
Z_{\mathrm{cl}}(\omega)=\frac{V_{\mathrm{cav}}(\omega)}{I_{b}(\omega)}=\frac{Z(\omega)}{1+e^{-i \tau \omega} G(\omega) Z(\omega) e^{i \phi}}
$$

where $\tau$ is the overall loop delay (including the klystron group delay), $\phi$ is the loop phase adjusted to get zero phase at the fundamental, and $G$ the gain of the llrf-generatorcoupler chain. Figure 3 shows an example of the open-loop impedance $Z(\omega)$ and the close-loop impedance $Z_{\mathrm{cl}}(\omega)$.

The feedback can reduce the impedance at frequencies where the product $G(\omega) Z(\omega)$ is significantly larger than 1 . The performance is limited by the group delay, a classic result with feedback systems. With a proportional feedback ( $G$ independent of $\omega$ ), after optimization of the llrf gain, the minimum value of the impedance seen by the beam is $[10]$

$$
R_{\min }=\frac{2}{\pi} \tau \frac{R}{Q} \omega_{r f}
$$

It is remarkable that this result does not depend on the cavity $Q_{L}$. It is proportional to the loop delay, as expected. To achieve high impedance reduction in the LHC, the distance between cavity-generator-llrf was kept short, with generator and llrf located in a cavern next to the machine tunnel. Care was also taken to minimize the llrf latency $(10 \mathrm{~ns})$ and the generator group delay (130 ns), resulting in a total loop delay of around $700 \mathrm{~ns}$. The minimum impedance is also proportional to the cavity $R / Q$. The LHC cavities were therefore designed for a low $R / Q$ (45 $\Omega$ ) [11]. The minimum impedance predicted by the simple formula (with proportional feedback) is about $50 \mathrm{k} \Omega$ per cavity. For comparison, the open-loop impedance is equal to $Q_{L} R / Q=0.9 \mathrm{M} \Omega$ for $Q_{L}=20000$ and $Q_{L} R / Q=2.7 \mathrm{M} \Omega$ for $Q_{L}=60000$. The LHC cavities have a single-cell geometry.

Figure 2 shows a block diagram of the implementation [12]. The rf feedback consists of three branches (i) The analog rf feedback is a low latency path with constant gain (proportional feedback) (ii) The digital rf feedback increases the gain by 10 (linear) in a very narrow (few hundreds of $\mathrm{Hz}$ ) band around the $\mathrm{rf}$ frequency. This provides very precise control of the static rf phase and voltage but has no effect on longitudinal stability, except for the mode zero where all bunches oscillate in phase (no effect at the revolution frequency sidebands). (iii) The oneturn feedback (OTFB) increases the gain by 10 (linear) in a 


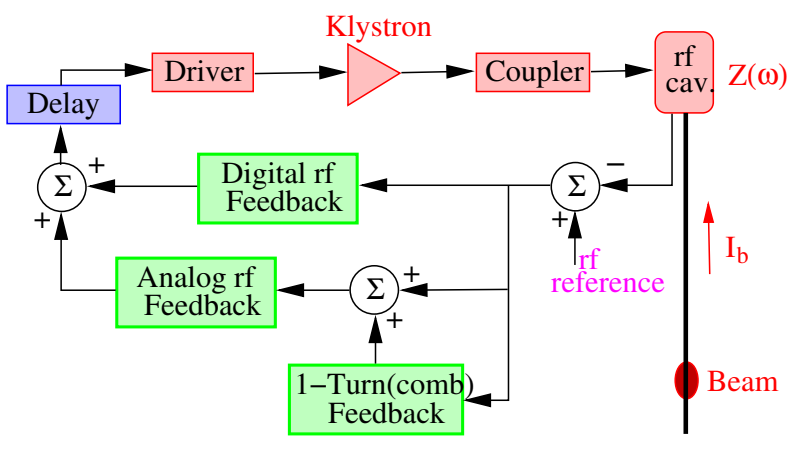

FIG. 2. Rf loop model block diagram with detailed llrf.

narrow band $(100 \mathrm{~Hz})$ on the revolution frequency sidebands [13]. It was first proposed for CERN's Super Proton Synchrotron (SPS), where the distance between generator and cavities did not allow for the use of a fast rf feedback [14]. More recently it was used in PEP-II, in tandem with a fast rf feedback [15] as in the LHC.

Figure 3 shows the reduction of the cavity impedance with the rf feedback (but no OTFB). The cavity is on tune, with $Q_{L}=20000$ (injection conditions). The analog feedback reduces the impedance by about $23 \mathrm{~dB}$ (14.1 linear). The digital feedback further reduces the impedance at the exact rf frequency by about $20 \mathrm{~dB}$ (10 linear). As explained above, the reduction is limited by the loop delay. Increasing the gain would distort the flat response and, if increased further, lead to loop instability. The achieved minimum impedance shown in Fig. 3 is $69 \mathrm{k} \Omega$, close to the $50 \mathrm{k} \Omega$ predicted from Eq. (2).

The OTFB further reduces the impedance, but only at the revolution frequency harmonics. Figure 4 shows the impedance with the OTFB, with an additional $20 \mathrm{~dB}$ reduction on the revolution frequency sidebands. The peak cavity

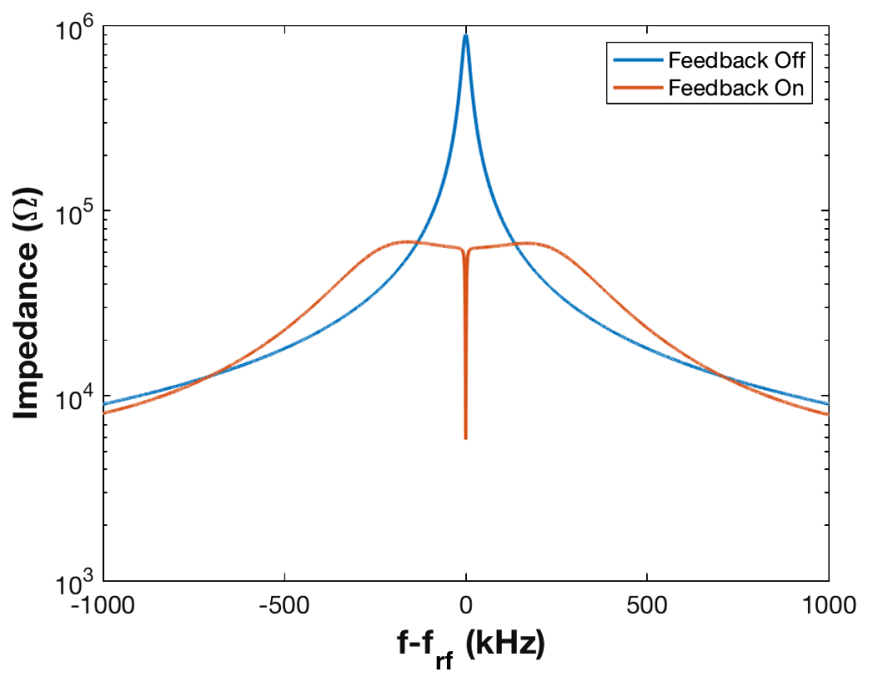

FIG. 3. Modulus of cavity impedance with and without rf feedback. Injection settings: $Q_{L}=20000, V_{c}=1 \mathrm{MV}$, cavity on tune.

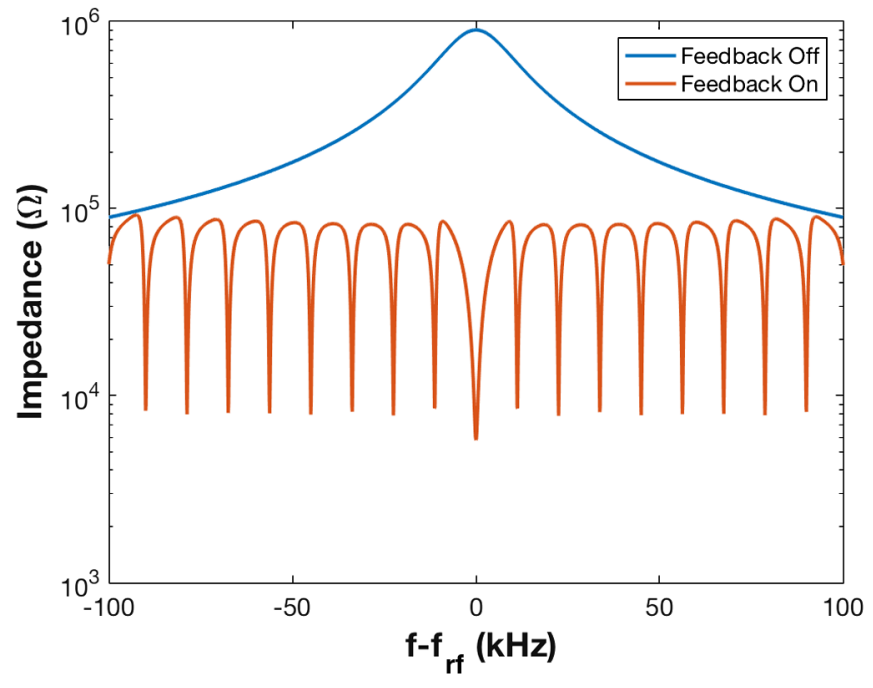

FIG. 4. Modulus of cavity impedance with OTFB. Injection settings: $Q_{L}=20000, V_{c}=1 \mathrm{MV}$, cavity on tune.

impedance is reduced from $0.9 \mathrm{M} \Omega$ (no feedback) to about $8 \mathrm{k} \Omega$ on the revolution frequency sidebands $\left(f_{\text {rev }}=11245 \mathrm{~Hz}\right)$.

Figure 5 shows the same result in physics $\left(Q_{L}=60000\right.$ [16]). The initial cavity impedance is three times higher $\left(2.7 \mathrm{M} \Omega\right.$ as it scales with $\left.Q_{L}\right)$, but the reduced impedance is the same. The llrf adjusts the gain tracking the main coupler position $\left(Q_{L}\right)$ so that the reduced impedance $(\approx 70 \mathrm{k} \Omega /$ cavity with OTFB off, about $9 \mathrm{k} \Omega$ /cavity with OTFB on) is only defined by the physical limitation due to the loop delay. The above figures are computed from an analytical expression for cavity and llrf processing. Data from the physical implementation will be shown below.

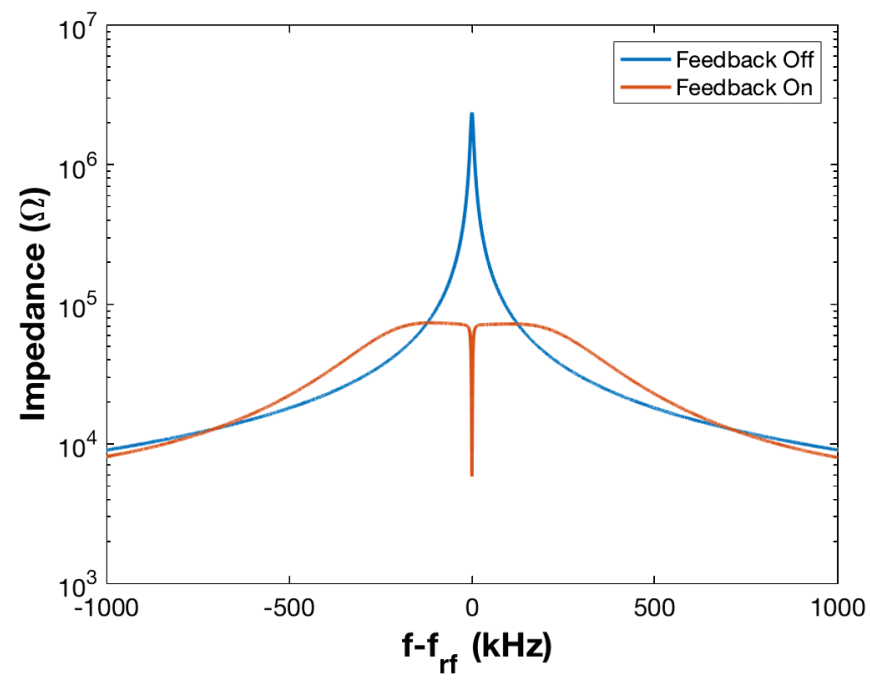

FIG. 5. Modulus of cavity impedance with and without $\mathrm{rf}$ feedback (OTFB off). Physics settings: $Q_{L}=60000$, $V_{c}=2 \mathrm{MV}$, cavity on tune. 
Models of the rf and llrf system [6,17] were developed before and during the LHC commissioning to offer insight on rf noise levels and sources, to study the rf effect on beam dynamics, to provide optimization and configuration tools for the LHC rf $[18,19]$, and to simulate the behavior of the rf loops with and without beam.

These linear reduced models provide an analytical representation of the estimated transfer function around a given operation point. The models include the accelerating super-conducting cavity, the $300 \mathrm{~kW}$ klystron and the llrf feedback (both digital and analog paths, as well as the OTFB). The waveguide, cable, and processing delays are included. The linearized model is parametrized by the few variables that could be adjusted during operation, including the rf feedback gain and phase, the cavity detuning and loaded quality factor $Q_{L}$, and more. The model follows the block diagram shown in Fig. 2.

During the annual commissioning of the LHC rf system and when adjustments are required during the LHC run, the closed loop transfer function of the rf station $H_{\text {meas }}(\omega)$ is measured by injecting noise in the loop. Then, the transfer function of the linearized model of the station, $H_{\text {model }}(\omega)$, is fitted to the measured function $H_{\text {meas }}(\omega)$ for the given operation point by adjusting the model's characteristic parameters. This parametrization of the transfer function allows the calculation of the open loop transfer function of the station around the actual operation point. With the open loop estimate, it is possible to determine the optimal values for the rf feedback settings. Through this model-based technique, the llrf systems of the physical machine are configured and studied over the range of high-current operating points, and the rf systems are periodically adjusted and "tuned" in operation.

Figure 6 shows an example of the measured and modeled open loop transfer function from cavity 1B1 in the LHC, whereas Figure 7 shows the closed loop case. To measure the
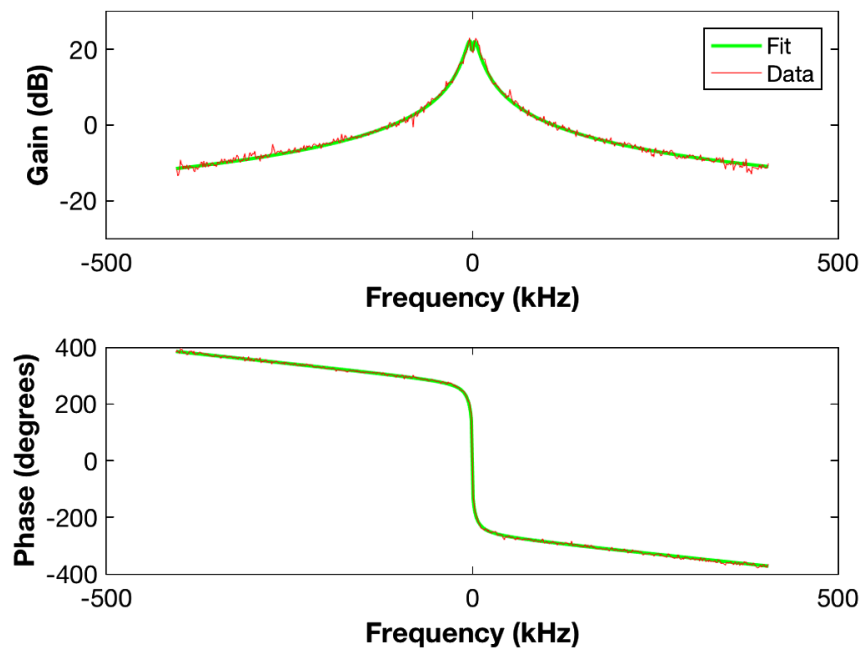

FIG. 6. LHC Cavity 1B1 measured and modeled open loop transfer function $\left(Q_{L}=20000\right)$.
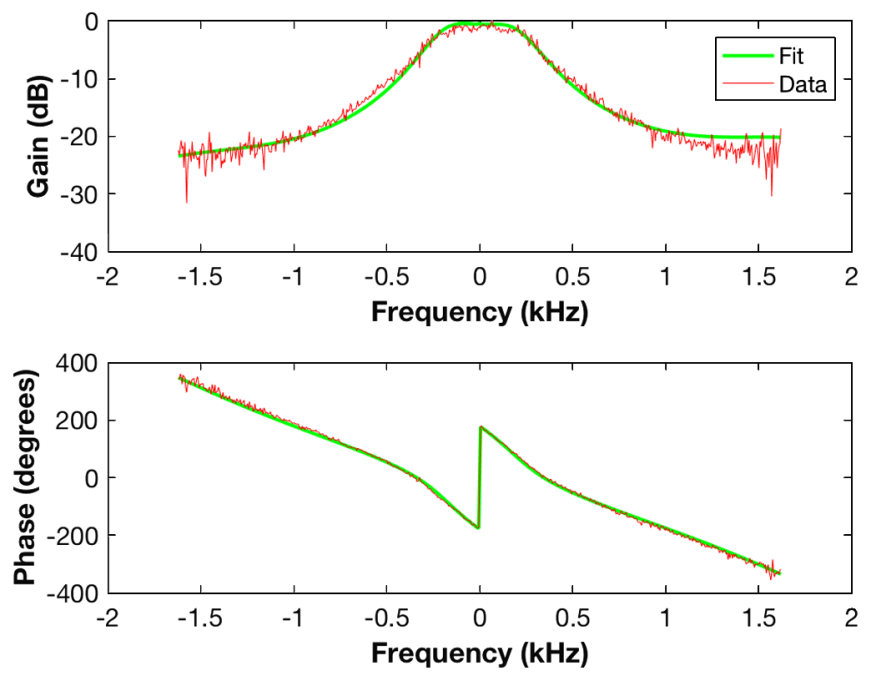

FIG. 7. LHC Cavity 1B1 measured and modeled closed loop transfer function $\left(Q_{L}=20000\right)$.

open-loop response, a switch at the llrf feedback output is opened, noise is injected right after the switch and the signal is measured right before the switch. The corresponding transfer function is $e^{-i \tau \omega} G(\omega) Z(\omega) e^{i \phi}$; a scaled version of the cavity impedance (for proportional feedback), with the linear phase shift caused by the system delay. In closed loop, the noise excitation is injected in the reference (Figs. 1, 2) and the cavity voltage is measured. The resulting transfer function is $e^{-i \tau \omega} G(\omega) Z(\omega) e^{i \phi} /\left[1+e^{-i \tau \omega} G(\omega) Z(\omega) e^{i \phi}\right]$. With a proportional feedback, this is a scaled version of the closed-loop cavity impedance [Eq. (1)], with the addition of the system delay and phase shift.

Comparing Fig. 3 to Figs. 6, 7 there is indeed a clear resemblance as they correspond to the same parameters (zero detuning, $Q_{L}=20000, V_{c}=1 \mathrm{MV}$ ). But Fig. 3 is an analytical calculation while Figs. 6, 7 present the parametric model fitted to measured data. This parametric model is used in the growth rate calculations.

\section{FUNDAMENTAL IMPEDANCE ESTIMATION}

The rf loop models are also a very useful tool in the estimation of the effective impedance [20] presented to the beam by the rf station, which strongly depends on the llrf configurations. Due to the close relationship between the models and the LHC rf configuration tools, it is straightforward to use the present llrf parameters from LHC and/or to optimize the rf model in exactly the same way as the real station. An advantage of this model-based approach is the ability to vary individual llrf feedback parameters and determine their effect on the beam stability. As a result, the sensitivity on individual rf parameters can be estimated, and the possible tradeoffs between beam and rf station stability can be investigated. The related results are presented in Sec. VII. 
Using the model estimated impedance, the growth rate $\sigma_{n}^{m}$ and tune shift $\Delta \omega_{n}^{m}$ can be computed for each coupledbunch mode $n$ and individual bunch oscillation mode $m$ ( $m=1$ for dipole, $m=2$ for quadrupole, etc.), assuming a parabolic line density and equispaced bunches [21]

$$
\begin{aligned}
\Lambda_{n}^{m}= & \sigma_{n}^{m}+j \Delta \omega_{n}^{m}=\frac{3}{2 \sqrt{\pi}} \frac{\Gamma\left(m+\frac{1}{2}\right)}{(m-1) !} \\
& \times \frac{M N r_{o} \eta c^{3}}{\gamma T_{o} \omega_{s} \hat{z}^{3}}\left(\frac{Z(\omega)}{\omega}\right)_{\mathrm{eff}} \\
= & \frac{3}{2 \sqrt{\pi 5^{3}}} \frac{\Gamma\left(m+\frac{1}{2}\right)}{(m-1) !} \frac{M N \eta q^{2}}{E_{o} T_{o} \omega_{s} \sigma_{\tau}^{3}}\left(\frac{Z(\omega)}{\omega}\right)_{\mathrm{eff}} \\
\left(\frac{Z(\omega)}{\omega}\right)_{\mathrm{eff}}= & \frac{\sum_{p=-\infty}^{\infty} \frac{Z(\omega)}{\omega} h_{m}(\omega)}{M \sum_{p=-\infty}^{\infty} h_{m}(\omega)}
\end{aligned}
$$

where the impedance is evaluated at frequencies $\omega=(p M+n) \omega_{o}+m \omega_{s}, \omega_{o}$ is the angular revolution frequency, $M$ is the number of equispaced bunches, $N$ are the particles per bunch, $r_{o}$ is the classical radius of the particle, $\eta=1 / \gamma_{t}^{2}-1 / \gamma^{2}$ is the slippage factor, $c$ is the speed of light, $\gamma$ is the Lorentz factor, $T_{o}$ is the revolution period, $\omega_{s}$ is the angular synchrotron frequency, $2 \hat{z}$ is the full bunch length $\left(\hat{z}=\sqrt{5} \sigma_{z}=\sqrt{5} c \sigma_{\tau}\right.$ for parabolic line density), $Z(\omega)$ is the estimated rf station impedance contributed from all 8 stations per beam, $q$ is the charge of a proton, $E_{o}$ is the beam energy, and $h_{m}(\omega)$ is the bunch mode spectral power density. For a parabolic distribution,

$$
h_{m}(\omega)=\frac{\left[J_{m+\frac{1}{2}}(|\omega \hat{z} / c|)\right]^{2}}{|\omega \hat{z} / c|}=\frac{\left[J_{m+\frac{1}{2}}\left(\left|\omega \sqrt{5} \sigma_{\tau}\right|\right)\right]^{2}}{\left|\omega \sqrt{5} \sigma_{\tau}\right|}
$$

where $J_{m}(x)$ is the Bessel function of the first kind.

In the LHC, the bunches are not equispaced as gaps are required for the injection and beam dump kickers rise time. Outside the gaps, the bunches are equispaced by $25 \mathrm{~ns}$ (10 $\mathrm{rf}$ periods). The maximum number of bunches is 2808 and the $3.5 \mu \mathrm{s} \mathrm{LHC}$ abort gap is much smaller than the revolution period of $89 \mu \mathrm{s}$. In this work, the above formula is used with $M=h / 10=3564$, corresponding to the equispaced situation of a full machine with $25 \mathrm{~ns}$ bunch spacing, and $2.2 \times 10^{11}$ protons per bunch $(h=35640$ is the harmonic number). The estimated growth rates presented in this work are thus elevated and the stability margins are conservative.

Figure 8 shows

$$
H_{m}(\omega)=\frac{\Gamma\left(m+\frac{1}{2}\right)}{(m-1) !} \frac{h_{m}(\omega)}{\sum_{p=-\infty}^{\infty} h_{m}(\omega)}
$$

The vertical line indicates the narrow range of $(\omega)$ values for the LHC based on the closed loop cavity bandwidth

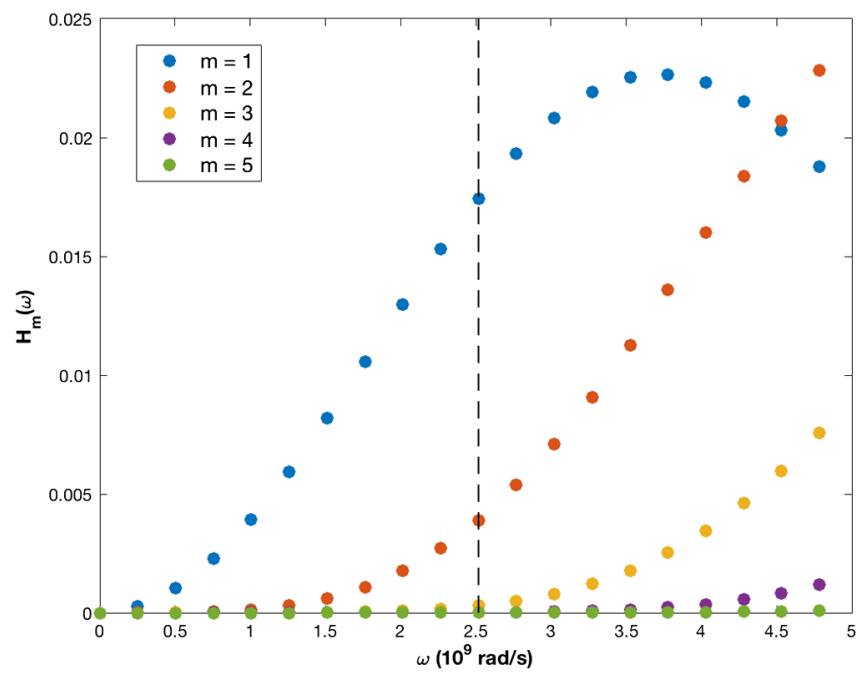

FIG. 8. Plot of $H_{m}(\omega)$ with $(\omega)$, for $p$ from 1 to 20 for $\sigma_{\tau}=250 \mathrm{ps}$.

( $400.8 \pm 0.4 \mathrm{MHz}$ ) for a $4 \sigma_{\tau}$ bunch duration of ( $\left.1 \mathrm{~ns}\right)$. It is clear that the dipole mode couples more strongly than any other bunch oscillation mode $m$. As presented in [22], the dipole mode couples most strongly when the bunch length is half the resonator period. The higher modes (quadrupole,..) will have smaller growth rates.

For a narrow-band accelerating cavity-a cavity with bandwidth much narrower than the inverse bunch spacing $M \omega_{o} / 2 \pi=40 \mathrm{MHz}$ in the LHC - there are only two terms that contribute to the summation ( $p M= \pm h$, where $h$ is the harmonic number, not to be confused with the bunch mode spectral power density $h_{m}(\omega)$ at $\omega_{1}=(h+n) \omega_{o}+m \omega_{s}$ and $\omega_{2}=(-h+n) \omega_{o}+m \omega_{s}$ since the $Z(\omega)$ impedance is negligible elsewhere. Therefore,

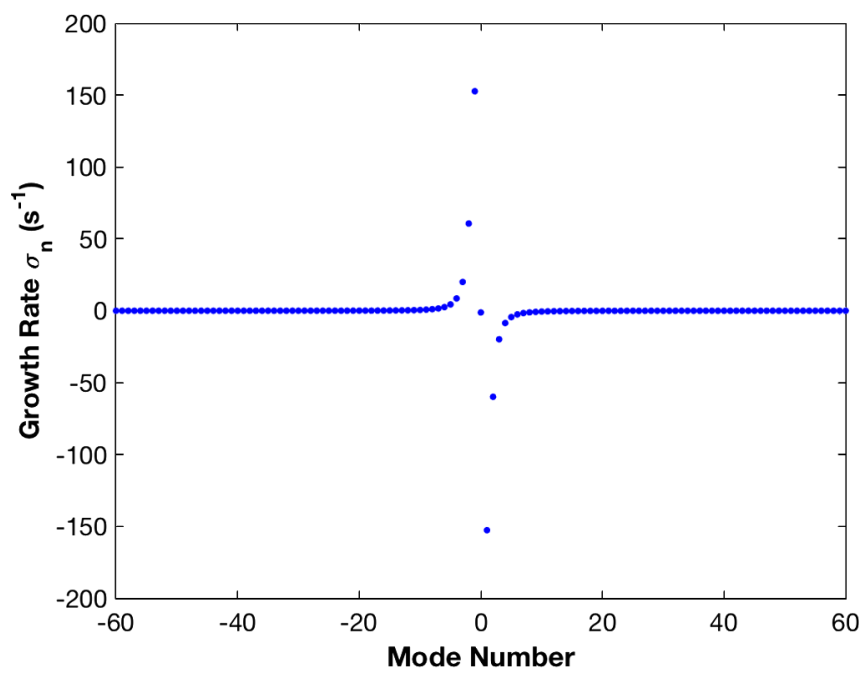

FIG. 9. Growth rates with mode number. Rf feedback off. $Q_{L}=20000$, Detuning $\Delta f=-10.4 \mathrm{kHz}$. 
TABLE I. Maximum value of $\left|\Lambda_{n}\right|$ over all $n$ caused by the impedance of the eight cavities at the fundamental. OTFB Off.

\begin{tabular}{lccccccc}
\hline \hline $\begin{array}{l}\text { Energy } \\
(\mathrm{GeV})\end{array}$ & $Q_{L}$ & $\begin{array}{c}\text { Detuning } \\
\text { scheme }\end{array}$ & $\begin{array}{c}\text { Detuning } \\
(\mathrm{kHz})\end{array}$ & $\begin{array}{c}\text { Total rf } \\
\text { Voltage }(\mathrm{MV})\end{array}$ & $\max (|\Lambda|) \mathrm{s}^{-1}$ & $\begin{array}{c}\text { Synchrotron } \\
\text { Frequency }(\mathrm{Hz})\end{array}$ & $\Delta \omega_{s} / 4\left(\mathrm{~s}^{-1}\right)$ \\
\hline 450 & 20000 & Half & -10.4 & 8 & 2.06 & 63.7 & 9.9 \\
450 & 20000 & Full & -16.3 & 8 & 3.21 & 63.7 & 9.9 \\
7000 & 60000 & Full & -8.2 & 16 & 0.36 & 23.0 & 3.6 \\
\hline \hline
\end{tabular}

$$
\begin{aligned}
{\left[\frac{Z(\omega)}{\omega}\right]_{\mathrm{eff}, m, n} } & =\frac{\frac{Z\left(\omega_{1}\right)}{\omega_{1}} h_{m}\left(\omega_{1}\right)+\frac{Z\left(\omega_{2}\right)}{\omega_{2}} h_{m}\left(\omega_{2}\right)}{M \sum_{p=-\infty}^{\infty} h_{m}(\omega)} \\
& \approx \frac{\left[\frac{Z\left(\omega_{1}\right)}{\omega_{1}}-\frac{\overline{Z\left(-\omega_{2}\right)}}{-\omega_{2}}\right] h_{m}\left(\omega_{1}\right)}{M \sum_{p=-\infty}^{\infty} h_{m}(\omega)}
\end{aligned}
$$

since the impedance is Hermitian and $h_{m}\left(\omega_{1}\right) \approx h_{m}\left(\omega_{2}\right)$. Notice that $-\omega_{2}=(h-n) \omega_{o}-m \omega_{s}$ so for mode $n$ the two contributions correspond to the $n^{\text {th }}$ upper and lower revolution sidebands. As a result, for an idling cavity ( $\mathrm{rf}$ feedback is off), only the low coupled-bunch mode numbers are within the cavity bandwidth and will have non-negligible growth rates, as shown in Fig. 9. In this case, mode zero is stable thanks to the detuning (Robinson stability [23]). Mode -1 is the most unstable, with a growth rate of $153 \mathrm{~s}^{-1}$, almost two orders of magnitude bigger than the maximum growth rate $\left(2.06 \mathrm{~s}^{-1}\right)$ in the presence of the rf feedback (Table I presented in Sec. VI).

When the rf feedback is on, the cavity impedance is replaced by $Z_{\mathrm{cl}}(\omega)$ [Eq. (1)] that has a lower peak value but now covers a much broader frequency band. The growth rates of the low order coupled-bunch modes are much lower but many more modes have to be considered now. In the presence of a well adjusted rf feedback system and for small

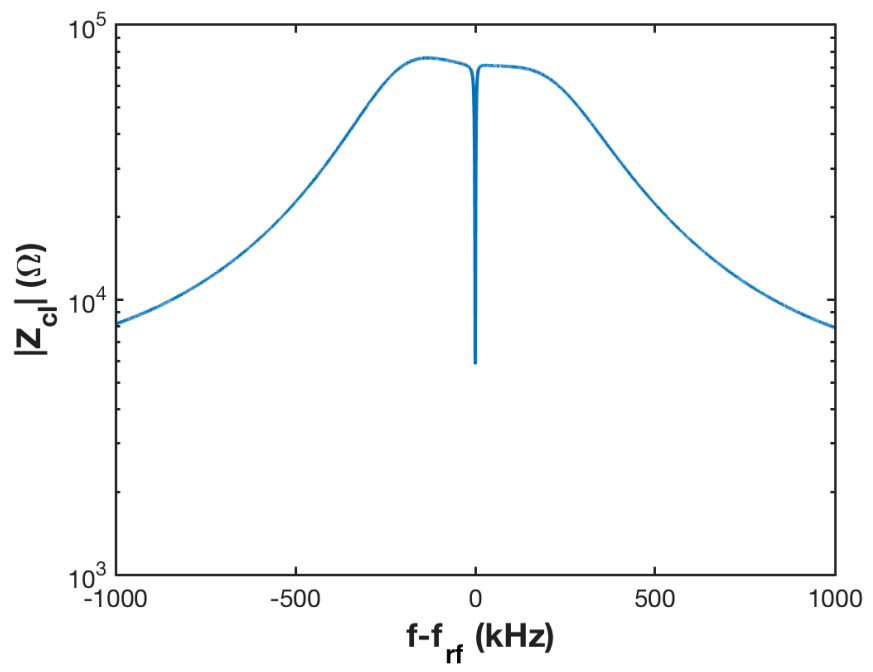

FIG. 10. Modulus of $Z_{c l}$. Physics settings: $Q_{L}=60000$, $V_{c}=2 \mathrm{MV}$, detuning of $-8.2 \mathrm{kHz}$. Rf station optimized with zero detuning. detuning the impedance $Z_{\mathrm{cl}}$ is Hermitian-symmetric with respect to $h \omega_{o}$ (Fig. 7). As a result, the real part of the contributions at $\omega_{1}$ and $-\omega_{2}$ mostly cancel, leading to a significant reduction of growth rates. However, in the presence of detuning, the impedances $Z_{\mathrm{cl}}$ at these two sidebands will no longer have equal amplitudes and the phases will not be exactly opposite, as shown in Figs. 10, 11. Therefore, the most unstable growth rate increases with detuning. Additionally, the impedance symmetry is sensitive to the rf feedback alignment and the cavity detuning. A sensitivity analysis is performed in Sec. VII.

\section{STABILITY THRESHOLD}

The interaction between the cavity fundamental impedance and the beam produces growth rates in the order of seconds when the llrf feedback system is operating, as will be shown in Sec. VI. Even though these growth rates are very slow - tens of thousands of turns - they are critical, because the longitudinal emittance synchrotron damping time is in the order of hours (approximately 50,000 hours for injection at $450 \mathrm{GeV}$ and 13 for physics at $7 \mathrm{TeV}$ ). For comparison, the synchrotron damping time at PEP-II was in the order of tens of milliseconds. Since there is no longitudinal feedback system, stability is determined by

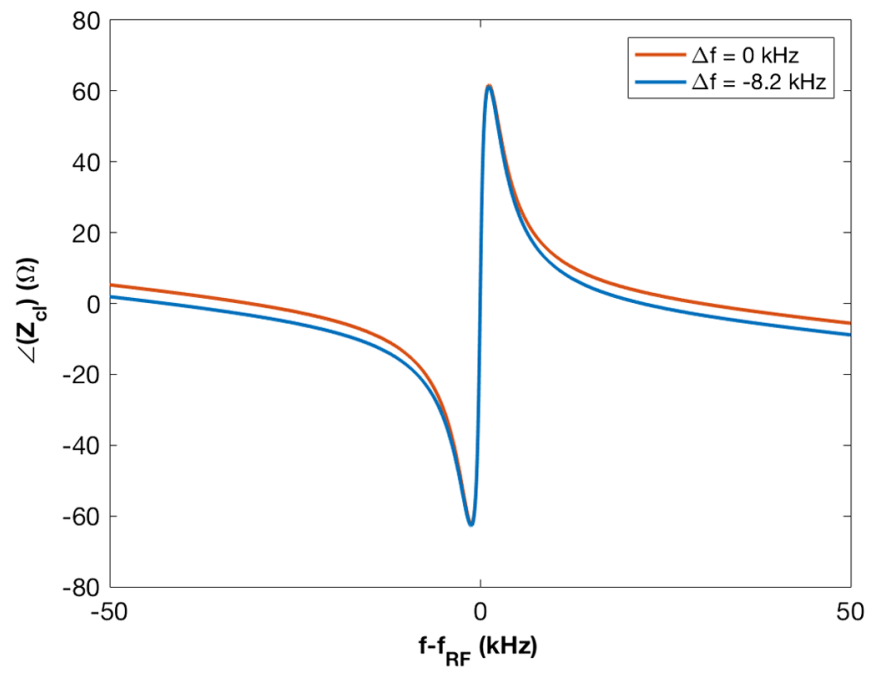

FIG. 11. $\quad Z_{\mathrm{cl}}$ phase for detuning of zero and $-8.2 \mathrm{kHz}$. Physics settings: $Q_{L}=60000, V_{c}=2 \mathrm{MV}$. Rf station optimized with zero detuning. 
Landau damping - a physical process which stabilizes the otherwise unstable ensemble of oscillating particles through a spread of their natural frequencies caused by the nonlinearity of the rf voltage. To determine stability, the simplified criterion defined in $[21,22]$ is utilized:

$$
\left|\Lambda_{n}^{m}\right|=\left|\sigma_{n}^{m}+j \Delta \omega_{n}^{m}\right|<\frac{\sqrt{m} \Delta \omega_{s}}{4}
$$

where $\Delta \omega_{s}$ is the synchrotron frequency spread within the bunch. In the present LHC with a single rf system, the spread in synchrotron frequency between center and edge of the bunch (total spread) is given by

$$
\Delta \omega_{s}=\omega_{s} \frac{\pi^{2}}{16}\left(\frac{h \hat{z}}{\pi R}\right)^{2}
$$

where $R=4242.893 \mathrm{~m}$ is the LHC radius.

As explained in the previous section, the dipole mode $(m=1)$ couples most strongly to the cavity impedance as shown in Fig. 8. Furthermore, the stability threshold increases as $\sqrt{m}$ and as a result, achieving stability for dipole bunch motion ensures stability for higher individual bunch oscillations modes (quadrupole, sextuple, etc.). As a result, the estimates presented in this paper correspond to the most critical case of the dipole mode $m=1$. The stability criterion can be simplified to

$$
\left|\Lambda_{n}\right|=\left|\sigma_{n}+j \Delta \omega_{n}\right|<\frac{\Delta \omega_{s}}{4} .
$$

\section{CONFIGURATIONS OF INTEREST}

The rf strategy for cavity detuning for high beam currents in the LHC has been summarized in [24]. At high beam currents, klystron forward power is expected to be the main rf limitation. For HiLumi LHC beams, the llrf will keep the rf voltage constant (amplitude and phase) over the turn, during filling. The cavity tuner will then employ the halfdetuning scheme, which minimizes the maximum klystron forward power over the beam/no-beam segments [25]. Before the ramp starts, the llrf will modulate the rf voltage in phase to follow the beam loading transients and the cavity tuner will be set to the full-detuning algorithm [24]. The half-detuning algorithm depends on the peak beam current (and thus the highest bunch charge), whereas the full detuning algorithm depends on the total beam current. Therefore, the ratio between the detuning values with the two algorithms is not equal to two.

In this work, the rf and beam parameters follow the latest HiLumi LHC specifications. There are three essential configurations with the HiLumi LHC intensity: $\alpha$ ) Half-detuning at $450 \mathrm{GeV}\left(V_{c}=1 \mathrm{MV}, Q_{L}=20000, \Delta f=-10.4 \mathrm{kHz}\right)$ $\beta)$ Full-detuning at $450 \mathrm{GeV}\left(V_{c}=1 \mathrm{MV}, Q_{L}=20000\right.$, $\Delta f=-16.3 \mathrm{kHz}) \gamma)$ Full-detuning at $7 \mathrm{TeV}\left(V_{c}=2 \mathrm{MV}\right.$, $Q_{L}=60000, \Delta f=-8.2 \mathrm{kHz}$ ) where $V_{c}$ is the cavity voltage, $Q_{L}$ is the cavity loaded $Q$, and $\Delta f$ is the cavity detuning. The crossing of the first revolution harmonic $\left(f_{\text {rev }}=11245 \mathrm{~Hz}\right)$ is also of interest. A bunch length of $1 \mathrm{~ns}\left(4 \sigma_{\tau}\right.$, corresponding to $1.12 \mathrm{~ns}$ full base width for a parabolic line density distribution) has been used in this work, consistent with the HiLumi parameters. The rf voltage could be changed though in operation to achieve different bunch length. Appendix explores the same configurations but for $0.75 \mathrm{MV}$ at $450 \mathrm{GeV}$ and $1.5 \mathrm{MV}$ at $7 \mathrm{TeV}$.

The LHC llrf is setup during the commissioning of each run. The optimal gain and phase settings for the feedback loops are determined without beam (zero detuning) for the planned $Q_{L}$ at injection and physics energy. As a result, in the presence of beam (resulting in reaction of the tuning system), the llrf settings slightly deviate from the optimal settings. This effect is taken into consideration by modeling a rf station optimized for zero detuning and by exploring the sensitivity to llrf settings in Sec. VII. In addition, the cavity detuning will be much higher in the HiLumi LHC than presently (higher beam current and full-detuning algorithm). To sustain the present $\mathrm{rf}$ station stability margins in the presence of significant detuning, the digital loop gain will be significantly reduced or the hardware will be modified. In both cases the impedance reduction around mode zero will be reduced. The digital loop gain has been set to zero in this work leading to conservative estimates of the stability margins.

\section{GROWTH RATE ESTIMATION}

A virtual rf station with zero detuning is first set up using the LHC rf configuration and optimization tools for $1 \mathrm{MV}$, $Q_{L}=20000$ and for $2 \mathrm{MV}, Q_{L}=60000$. The detuning is then varied for each configuration of interest. The impedance is estimated and scaled for the cumulative effect of

TABLE II. Maximum value of $\left|\Lambda_{n}\right|$ over all $n$ caused by the impedance of the eight cavities at the fundamental. OTFB On.

\begin{tabular}{lccccccc}
\hline \hline Energy $(\mathrm{GeV})$ & $Q_{L}$ & $\begin{array}{c}\text { Detuning } \\
\text { scheme }\end{array}$ & $\begin{array}{c}\text { Detuning } \\
(\mathrm{kHz})\end{array}$ & $\begin{array}{c}\text { Total } \mathrm{rf} \\
\text { Voltage }(\mathrm{MV})\end{array}$ & $\max (|\Lambda|) \mathrm{s}^{-1}$ & $\begin{array}{c}\text { Synchrotron } \\
\text { Frequency }(\mathrm{Hz})\end{array}$ & $\Delta \omega_{s} / 4\left(\mathrm{~s}^{-1}\right)$ \\
\hline 450 & 20000 & Half & -10.4 & 8 & 0.186 & 63.7 & 9.9 \\
450 & 20000 & Full & -16.3 & 8 & 0.196 & 63.7 & 9.9 \\
7000 & 60000 & Full & -8.2 & 16 & 0.025 & 23.0 & 3.6 \\
\hline \hline
\end{tabular}




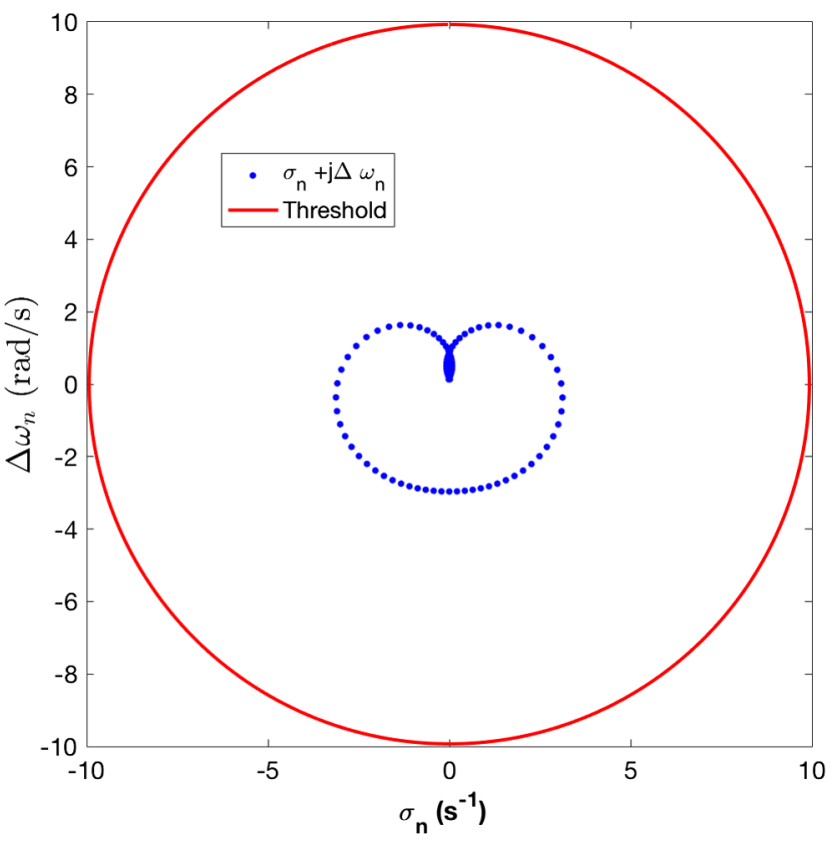

FIG. 12. $\Lambda_{n}=\sigma_{n}+j \Delta \omega_{n}$ and stability threshold. Configuration $(\beta)$, OTFB Off.

eight cavities per ring. Finally, the maximum value of $\left|\Lambda_{n}\right|$ over all $n$ in the bandwidth of the rf station and the stability threshold $\Delta \omega_{s} / 4$ are calculated and are reported in Table I for each configuration with the OTFB off. Table II shows the same configurations, but with the OTFB on. Figure 12 shows $\Lambda_{n}$ with the stability threshold for the most critical case of configuration $(\beta)$.

It is obvious from these two tables that even with the OTFB off there are considerable margins (more than a factor of three for the worst situation). It is also evident that the configurations at physics have lower $\Lambda_{n}$ values than

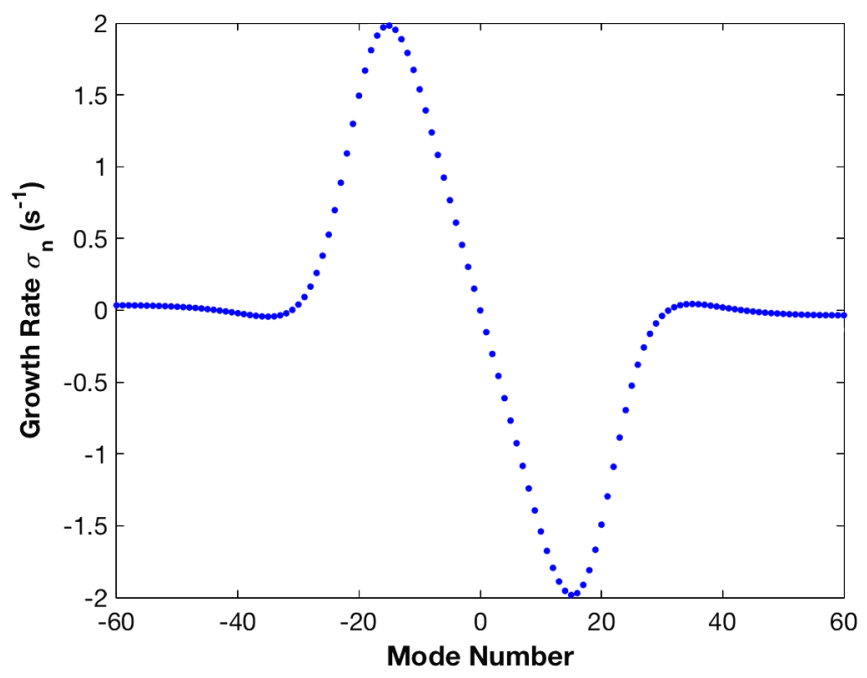

FIG. 13. Growth rates with mode number. Configuration $(\alpha)$, OTFB Off.

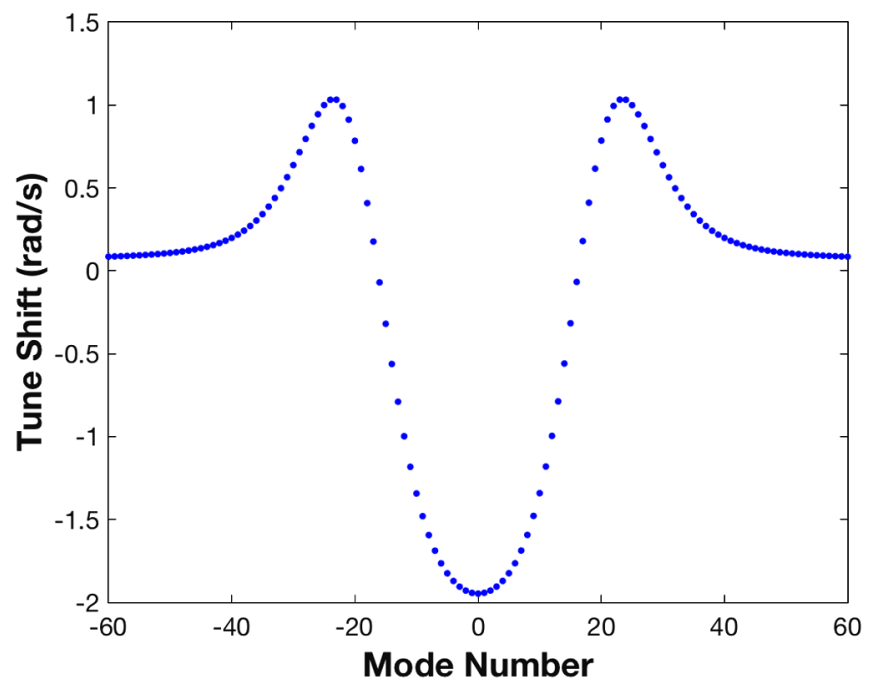

FIG. 14. Tune shift with mode number. Configuration $(\alpha)$, OTFB Off.

those at injection due to the almost twenty-fold increase in beam energy. In the LHC, longitudinal emittance blow-up keeps the bunch length constant during the acceleration, resulting in improved stability at high energy $[26,27]$. It is also evident that configuration $(\beta)$ is less stable than configuration $(\alpha)$ since the cavity detuning is bigger. Since the stability margins are so significant, the small dependence of the dipole mode stability thresholds on the longitudinal line density are not investigated in this work.

Figure 13 shows the resulting growth rates for each mode for configuration $(\alpha)$, whereas Fig. 14 shows the corresponding tune shifts.

\section{GROWTH RATE SENSITIVITY TO LLRF PARAMETERS}

The llrf system is setup to achieve specific rf loop stability criteria. These settings though are not necessarily optimal for beam stability considerations, or equivalently for maximum effective impedance reduction. These tradeoffs were observed at PEP-II and their study helped increase the stored beam current [5]. To better understand the importance of the llrf setup choices and the necessity for studies of rf loop and beam stability trade-offs, it is essential to analyze the sensitivity of $\Lambda_{n}$ to variations of the llrf parameters. These studies provide insight on the limits of the implementation, on the operational margins, and on the parameters most essential to reliable operation. A similar analysis was conducted for the first LHC run with very reassuring results [6].

Configuration $(\beta)$ at the end of flat bottom is used as a reference, since it presents the least stable situation. Each of the following parameters were modified separately to understand their impact in the interaction between the $\mathrm{rf}$ station and the beam dynamics: cavity detuning $\Delta f$, analog/digital loop gain $G$, controller phase $\phi$, and 
TABLE III. Highest $\left|\Lambda_{n}\right|$ Sensitivity on llrf parameters.

\begin{tabular}{lccc}
\hline \hline Parameter & Adjustment & $\max \left(\left|\Lambda_{n}\right|\right)\left(\mathrm{s}^{-1}\right)$ & Change \\
\hline$\Delta f$ & $\pm 3 \mathrm{kHz}$ & $2.61 / 3.80$ & $-19 /+18 \%$ \\
$G$ & $\pm 3 \mathrm{~dB}$ & $3.12 / 5.47$ & $-3 /+\mathbf{7 0} \%$ \\
$\phi$ & $\pm 5^{\circ}$ & $5.43 / 2.06$ & $+\mathbf{6 9} /-\mathbf{3 6} \%$ \\
$Q_{L}$ & $\pm 10 k$ & $3.35 / 2.83$ & $+4 /-12 \%$ \\
\hline \hline
\end{tabular}

cavity $Q_{L}$. The adjustments for each case correspond to reasonable variations over an LHC run. The system's impedance and corresponding $\Lambda_{n}$ values were estimated for each case. The OTFB was off to accentuate the effects. The maximum $\Lambda_{n}$ value for each case is reported in Table III.

As expected, there is considerable beam stability dependence on the controller gain and phase. A gain reduction increases $Z_{\mathrm{cl}}$ significantly, whereas a change in phase leads to an asymmetry in $Z_{\mathrm{cl}}$. Figures 15,16 show the highest $\left|\Lambda_{n}\right|$ as the llrf gain or phase are modified from the operational value. As was mentioned above, the operational values are chosen to achieve predetermined gain and phase margins for the rf loop, but they are not necessarily optimal in terms of beam stability. A trade-off exists between $\mathrm{rf}$ loop and beam stability, which could be explored if the beam stability margins are not sufficient.

This analysis also shows the critical importance of careful tuning of the llrf in cases where the beam stability margin is limited. A phase rotation of about $13^{\circ}$ is sufficient to make the beam unstable. On the other hand, it should be noted that this is the worse case scenario in terms of detuning and the OTFB is assumed to be off. In actual operation with the OTFB on, the rotation needed to reach instability is so high, that the rf loop would become unstable first.

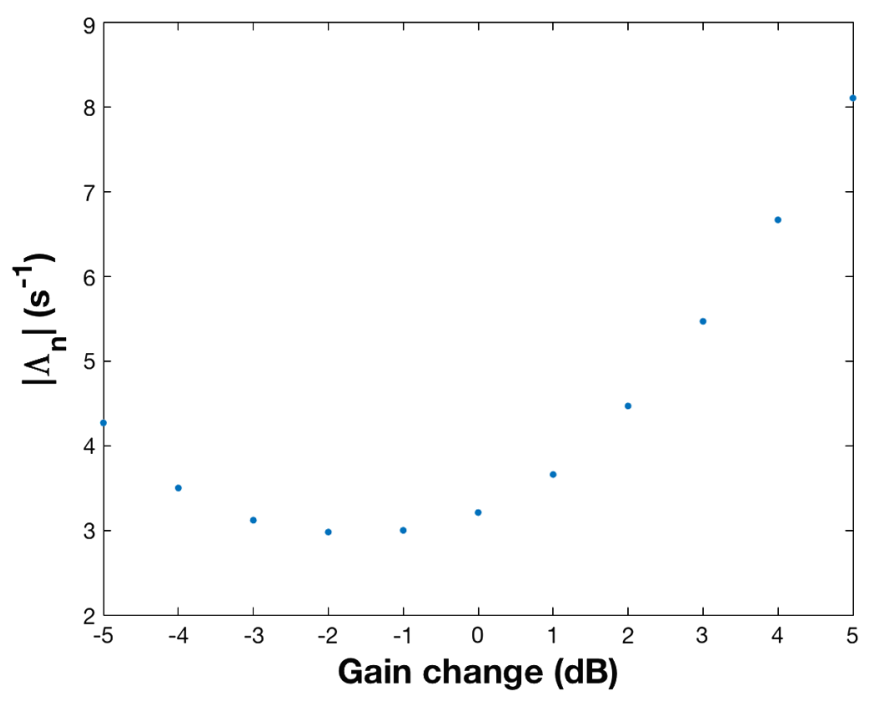

FIG. 15. Highest $\left|\Lambda_{n}\right|$ with llrf gain change. Configuration $(\beta)$, OTFB Off.

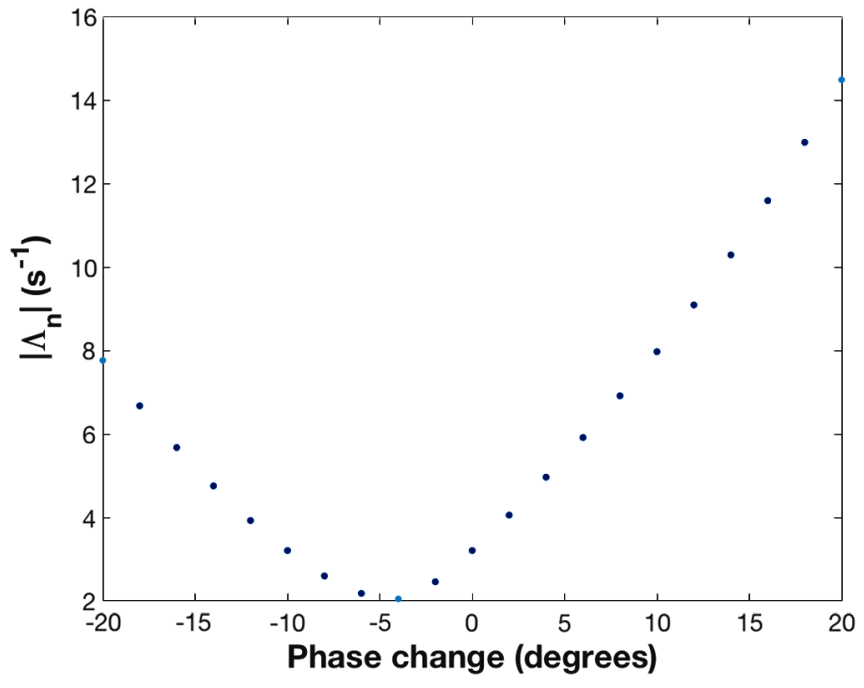

FIG. 16. Highest $\left|\Lambda_{n}\right|$ with llrf phase change. Configuration $(\beta)$, OTFB Off.

The sensitivity on the OTFB parameters was also estimated. The OTFB loop gain $G_{c}$ and phase $\phi_{c}$ were varied and the delay $\tau_{d}$ changed from the exact one-turn value. The maximum $\Lambda_{n}$ value for each case is reported in Table IV. Once again, there is a significant dependence of $\Lambda_{n}$ on the OTFB phase setting, reinforcing the need for careful llrf configuration and adjustment.

It is not surprising that there are changes in llrf parameters that improve beam stability. The llrf is tuned in a manner that balances the rf/llrf loop stability and the beam stability. The cavity detuning $\Delta f$ is set to minimize the average klystron power. The analog/digital loop gain as well as the OTFB loop gain are set to achieve predetermined gain margins. Therefore, a trade-off exists between beam and loop stability. For example, even though there were no observable effects on the estimated $\Lambda_{n}$ values with $\tau_{d}$ variations, a shift of even a few tens of nanoseconds is sufficient to bring rf station instability. Thus, optimal tuning of the OTFB delay might not be critical for beam stability directly, but it is essential for rf station stability, and consequently for reliable operation with beam. Similarly at PEP-II, it was determined that a few degrees phase rotation in the analog or OTFB feedback systems would significantly improve the beam stability with very small reduction of the llrf loop stability margins. These adjustments allowed much higher stored beam currents [5]. But

TABLE IV. Highest $\left|\Lambda_{n}\right|$ Sensitivity on llrf parameters.

\begin{tabular}{lccc}
\hline \hline Parameter & Adjustment & $\max \left(\left|\Lambda_{n}\right|\right)\left(\mathrm{s}^{-1}\right)$ & Change \\
\hline$G_{c}$ & $\pm 3 \mathrm{~dB}$ & $0.159 / 0.249$ & $-19 /+28 \%$ \\
$\phi_{c}$ & $\pm 10^{\circ}$ & $0.863 / 0.688$ & $+\mathbf{3 4 2} /+\mathbf{2 5 2} \%$ \\
$\tau_{d}$ & $\pm 100 \mathrm{~ns}$ & $0.196 / 0.196$ & $0 / 0 \%$ \\
\hline \hline
\end{tabular}


TABLE V. Maximum value of $\left|\Lambda_{n}\right|$ over all $n$ caused by the impedance of the eight cavities at the fundamental. OTFB Off. Rf station optimized with beam.

\begin{tabular}{lccccccc}
\hline \hline Energy $(\mathrm{GeV})$ & $Q_{L}$ & $\begin{array}{c}\text { Detuning } \\
\text { scheme }\end{array}$ & $\begin{array}{c}\text { Detuning } \\
(\mathrm{kHz})\end{array}$ & $\begin{array}{c}\text { Total } \mathrm{rf} \\
\text { Voltage }(\mathrm{MV})\end{array}$ & $\max (|\Lambda|) \mathrm{s}^{-1}$ & $\begin{array}{c}\text { Synchrotron } \\
\text { Frequency }(\mathrm{Hz})\end{array}$ & $\Delta \omega_{s} / 4\left(\mathrm{~s}^{-1}\right)$ \\
\hline 450 & 20000 & Half & -10.4 & 8 & $1.17(-43 \%)$ & 63.7 & 9.9 \\
450 & 20000 & Full & -16.3 & 8 & $2.03(-36 \%)$ & 63.7 & 9.9 \\
7000 & 60000 & Full & -8.2 & 16 & $0.19(-47 \%)$ & 23.0 & 3.6 \\
\hline \hline
\end{tabular}

such a fine optimization does not appear necessary in the LHC.

\section{A. Detuning crossing $f_{\text {rev }}$}

During injection, the cavity detuning is very close to and could cross the $f_{\text {rev }}=11245 \mathrm{~Hz}$ line. The $\left|\Lambda_{n}\right|$ values were estimated for a rf station at $450 \mathrm{GeV}$ with a detuning of 11 , 11.245 , and $11.5 \mathrm{kHz}$ and leading to growth rates of 2.17 , 2.22, and $2.27 \mathrm{~s}^{-1}$ respectively (OTFB Off). Therefore, no issues of longitudinal instability are expected when the cavity detuning crosses the $f_{\text {rev }}$ line. With the strong llrf feedback the cavity impedance is flattened and thus covers several revolution frequency lines (Fig. 3). As a result, the crossing of the cavity resonance has no significant effect.

\section{B. Optimizing the llrf system in the presence of beam}

Since the rf station is optimized without beam and then the cavity gets detuned when the beam is injected, the achieved impedance reduction is not optimal. A llrf optimization in the presence of beam is not trivial though. The present setting-up tools rely on transfer function measurements done by injecting noise in the llrf loops. This is clearly not desirable with a circulating beam. However, from a measurement of beam current, one could estimate the actual detuning and apply a feedforward correction on the llrf settings. A study was conducted to estimate the instability growth rates with an optimized rf station in the presence of beam. Table $\mathrm{V}$ presents the results. It is evident that optimizing the llrf station with beam, or equivalently in the presence of cavity detuning, leads to a significant reduction of the instability growth rates. This technique could be developed to further increase the stability margins.

\section{BEAM CURRENT THRESHOLD ESTIMATION}

The current threshold due to instabilities driven by the cavity fundamental was also estimated. Due to the detuning change with current, the highest $\left|\Lambda_{n}\right|$ is not simply linear with current. Instead, the beam current was slowly increased, the beam detuning was adjusted for the higher current, and the highest $\left|\Lambda_{n}\right|$ was estimated, until the stability threshold was crossed. In the presence of the OTFB, the DC current threshold is more than $17 \mathrm{~A}$, or more than 15 times the design parameter of the HiLumi LHC. This estimate does not include the llrf optimization in the presence of beam. The optimization would further push the DC current threshold to more than 22 A. Of course, single-bunch stability thresholds, power limitations, heating issues, and more will limit the maximum HiLumi LHC current to a much lower value. The thresholds above are just further evidence of the significant operational margin with regards to cavity fundamental driven coupled-bunch instabilities.

\section{GROWTH RATE SENSITIVITY TO BUNCH LENGTH}

From Eq. (3), the instability growth rates depend directly on $1 / \sigma_{\tau}^{3}$ and on the effective impedance. The effective impedance can be shown to have a $\sigma_{\tau}^{2}$ scaling around the LHC operational point. Therefore, the instability growth rates approximately scale with $1 / \sigma_{\tau}$. Additionally, the stability threshold scales as $\sigma_{\tau}^{2}$ (Eq.(4)). Therefore, the stability margin approximately increases with $\sigma_{\tau}^{3}$. The minimum bunch length to prevent coupled-bunch instabilities due to the fundamental cavity impedance can then be estimated to be $0.69 \mathrm{~ns}\left(4 \sigma_{\tau}\right)$ by scaling the stability margin and bunch length from Sec. VI for the least stable configuration (Table I). The bunch length at the end of the injection plateau has a standard deviation of up to $60 \mathrm{ps}$ [27] over all the bunches, so the $4 \sigma_{\tau}$ bunch length should be kept above the threshold plus 3 standard deviations $(\approx 0.87 \mathrm{~ns})$ to avoid unstable bunches in the absence of the OTFB.

The intensity threshold scales as $\sigma_{\tau}^{5}$ for single bunch instabilities. Based on measurements in the LHC, the single-bunch instability threshold is $0.94 \mathrm{~ns}$ [28] for the HiLumi LHC parameters and with the present machine impedance. Therefore, single-bunch effects are limiting the bunch length before the coupled-bunch instabilities due to the cavity fundamental impedance. This conclusion is supported by observations during the 2015 LHC run with 2200 bunches and an intensity of $1.1 \times 10^{11}$ protons per bunch. The bunch length reduced due to synchrotron radiation damping and after 20 hours of fill, it reached the figure corresponding to the single-bunch instability threshold measured during machine development (MD) sessions. Longitudinal instabilities were then observed [29]. 


\section{MEASUREMENTS OF LONGITUDINAL COUPLED-BUNCH INSTABILITIES}

An MD was conducted on October 6, 2016 to validate the above estimates and prove that longitudinal coupledbunch instabilities due to the cavity fundamental impedance are truly not an issue for higher beam currents. The MD was conducted with 2220 bunches in the LHC, of approximately $1.1 \mathrm{e} 11$ protons per bunch $(\approx 0.44$ A DC). The total rf voltage was set at $6 \mathrm{MV}$ and the cavity $Q_{L}$ to 20000. The cavity tuner was set to the half-detuning scheme. The MD was conducted at $450 \mathrm{GeV}$, since the longitudinal coupled-bunch instabilities growth rates are higher at that energy for a given bunch length. During the $\mathrm{MD}$, the fundamental cavity impedance was gradually increased by in order switching the OTFB off, reducing the llrf feedback gain, and finally rotating the rf loop phase in steps of $10^{\circ}$. As shown in Fig. 16, the rf loop phase has a strong effect on the maximum value of $\left|\Lambda_{n}\right|$ and thus on the beam stability.

As indicated in the previous section, the stability threshold scales with $\sigma_{\tau}^{3}$. As a result, the bunch length is a very reliable metric for the accuracy of our models. Figure 17 shows the measured bunch length and the estimated threshold using our model. The threshold steps correspond to two steps in the rf loop phase of $10^{\circ}$ each. It is clear from this image that an instability ensued whenever the threshold was crossed, which led to oscillations around the synchronous position. The resulting filamentation leads to bunch lengthening, which finally restores stability. In-between the two instability instances the most unstable modes were excited by injecting a signal with the appropriate spectral content in the rf cavities. This excitation led to the smaller bunch length increases. These are not due to instabilities, but rather the result of the resonant excitation. Finally, the slow constant growth is a result of intrabeam scattering.

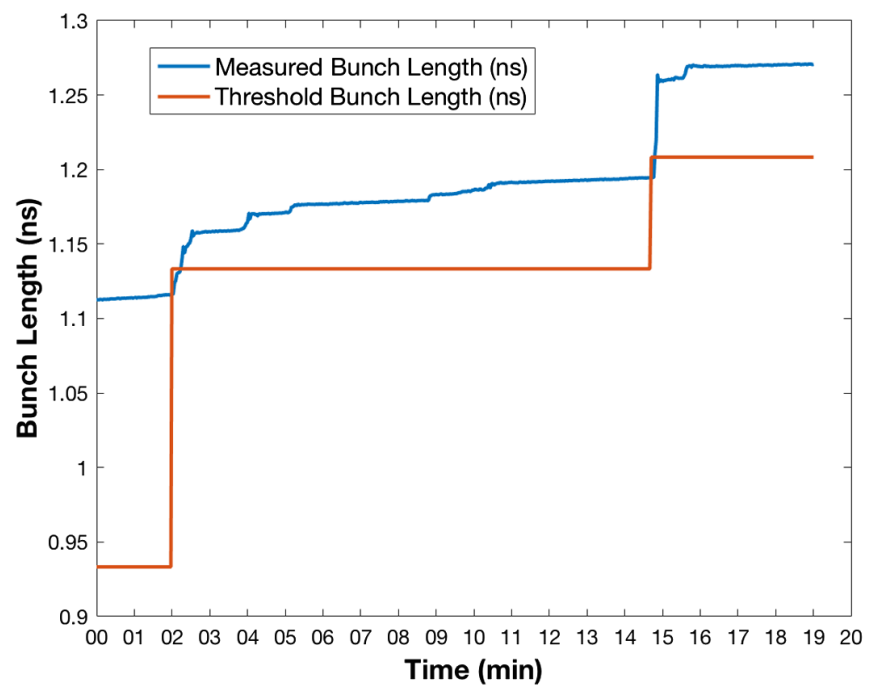

FIG. 17. Measured and threshold $4 \sigma_{\tau}$ bunch length (ns).
TABLE VI. Bunch length before and after instability, and corresponding threshold estimate.

\begin{tabular}{lcc}
\hline \hline Initial $4 \sigma_{\tau}(\mathrm{ns})$ & Final $4 \sigma_{\tau}(\mathrm{ns})$ & Threshold Estimate $(\mathrm{ns})$ \\
\hline 0.98 & 1.28 & 1.13 \\
1.00 & 1.18 & 1.13 \\
1.11 & 1.16 & 1.13 \\
1.12 & 1.18 & 1.13 \\
1.18 & 1.29 & 1.20 \\
1.19 & 1.26 & 1.20 \\
\hline \hline
\end{tabular}

This procedure was repeated twice for each of the two beams in the LHC, resulting to seven instability instances. Table VI summarizes the bunch length before the llrf parameter change, the bunch length when stability is restored, and the estimated threshold corresponding to the modified llrf settings. This table shows that the model predicts the stability threshold fairly accurately, even with the very sensitive threshold dependence on $\sigma_{\tau}^{3}$. The final bunch length is significantly higher than the threshold since the bunch length still increases while the oscillations are slowly damped after stability is restored.

\section{CONCLUSIONS}

Longitudinal coupled-bunch instability growth rates due to the cavity fundamental impedance were estimated for LHC beams in the HiLumi era and for realistic cavity configurations using the experience from LHC operation. Significant stability margins are anticipated with the HiLumi beam, even with the OTFB off. As a result, it is not necessary to explore the tradeoff between rf station and beam stability. The sensitivity to llrf parameters was investigated, suggesting a large dependence of the longitudinal growth rates on the cavity controller and OTFB phase. Even a $10^{\circ}$ variation of these phases would not lead to an unstable beam though. The situation when the cavity detuning crosses the revolution frequency was also investigated. No critical situations for beam stability were discovered in this case either. Initial measurements confirming the significant stability margin were also presented. The stability margin is strongly dependent on the bunch length though and operation with a mean bunch length of less than 0.87 ns would lead to unstable bunches, in the absence of OTFB. With the OTFB, an additional factor of ten in stability margin is gained. Therefore, coupled-bunch instabilities driven by the cavity fundamental impedance should not be an issue for the HiLumi LHC.

\section{ACKNOWLEDGMENTS}

The authors would like to thank Elena Shaposhnikova for useful discussions on stability thresholds, Juan Muller for access to MD data, Gianluigi Arduini and Elias Metral for improving this work through discussions and suggested improvements, and the LHC OP group for assistance 
TABLE VII. Maximum value of $\left|\Lambda_{n}\right|$ over all $n$ caused by the impedance of the eight cavities at the fundamental. OTFB Off.

\begin{tabular}{lccccccc}
\hline \hline Energy $(\mathrm{GeV})$ & $Q_{L}$ & $\begin{array}{c}\text { Detuning } \\
\text { scheme }\end{array}$ & $\begin{array}{c}\text { Detuning } \\
(\mathrm{kHz})\end{array}$ & $\begin{array}{c}\text { Total } \mathrm{rf} \\
\text { Voltage }(\mathrm{MV})\end{array}$ & $\max (|\Lambda|) \mathrm{s}^{-1}$ & $\begin{array}{c}\text { Synchrotron } \\
\text { Frequency }(\mathrm{Hz})\end{array}$ & $\Delta \omega_{s} / 4\left(\mathrm{~s}^{-1}\right)$ \\
\hline 450 & 20000 & Half & -13.8 & 6 & 3.13 & 55.2 & 8.6 \\
450 & 20000 & Full & -21.8 & 6 & 4.95 & 55.2 & 8.6 \\
7000 & 60000 & Full & -10.9 & 12 & 0.55 & 19.9 & 3.1 \\
\hline \hline
\end{tabular}

TABLE VIII. Maximum value of $\left|\Lambda_{n}\right|$ over all $n$ caused by the impedance of the eight cavities at the fundamental. OTFB On.

\begin{tabular}{lccccccc}
\hline \hline Energy $(\mathrm{GeV})$ & $Q_{L}$ & $\begin{array}{c}\text { Detuning } \\
\text { scheme }\end{array}$ & $\begin{array}{c}\text { Detuning } \\
(\mathrm{kHz})\end{array}$ & $\begin{array}{c}\text { Total rf } \\
\text { Voltage }(\mathrm{MV})\end{array}$ & $\max (|\Lambda|) \mathrm{s}^{-1}$ & $\begin{array}{c}\text { Synchrotron } \\
\text { Frequency }(\mathrm{Hz})\end{array}$ & $\Delta \omega_{s} / 4\left(\mathrm{~s}^{-1}\right)$ \\
\hline 450 & 20000 & Half & -13.8 & 6 & 0.206 & 55.2 & 8.6 \\
450 & 20000 & Full & -21.8 & 6 & 0.223 & 55.2 & 8.6 \\
7000 & 60000 & Full & -10.9 & 12 & 0.029 & 19.9 & 3.1 \\
\hline \hline
\end{tabular}

during MD sessions. The HiLumi LHC Design Study is included in the High Luminosity LHC project and is partly funded by the European Commission within the Framework Programme 7 Capacities Specific Programme, Grant Agreement 284404. This work is also partially supported by the National Science Foundation under Grant No. PHY1535536.

\section{APPENDIX: ALTERNATIVE rf OPERATIONAL CONFIGURATIONS}

The rf could be operated with a lower voltage in the HiLumi LHC era to achieve different longitudinal emittance, without changing the nominal bunch length. The possible scenarios of operation with $750 \mathrm{kV}$ per cavity (6 MV total) at $450 \mathrm{GeV}$ and $1.5 \mathrm{MV}$ per cavity (12 MV total) at $7 \mathrm{TeV}$ was investigated. The resulting growth rates are shown in Tables VII and VIII in the absence and presence of the OTFB, showing a small but not concerning increase in growth rates with these operational settings.

[1] D. Boussard, D. Brandt, and L. Vos, CERN Technical Report No. LHC-PROJECT-NOTE-205, 1999.

[2] The LHC cavities are equipped with movable power couplers: the machine is filled with $Q_{L}=20000$, then the coupler is retracted to a position corresponding to $Q_{L}=60000$ during ramping and physics.

[3] J. D. Fox, T. Mastorides, C. Rivetta, D. Van Winkle, and D. Teytelman, Lessons learned from positron-electron project low level rf and longitudinal feedback, Phys. Rev. ST Accel. Beams 13, 052802 (2010).
[4] T. Mastorides, C. Rivetta, J. D. Fox, D. Van Winkle, and D. Teytelman, Analysis of longitudinal beam dynamics behavior and rf system operative limits at high-beam currents in storage rings, Phys. Rev. ST Accel. Beams 11, 062802 (2008).

[5] C. Rivetta, T. Mastorides, J. D. Fox, D. Teytelman, and D. Van Winkle, Modeling and simulation of longitudinal dynamics for low energy ring - high energy ring at the positron-electron project, Phys. Rev. ST Accel. Beams 10, 022801 (2007).

[6] T. Mastorides, C. Rivetta, J. D. Fox, D. V. Winkle, and P. Baudrenghien, rf system models for the CERN Large Hadron Collider with application to longitudinal dynamics, Phys. Rev. ST Accel. Beams 13, 102801 (2010).

[7] The beam loading factor is defined as the ratio of the $\mathrm{rf}$ component of the beam current (around $2 \mathrm{~A}$ for HiLumi parameters) to the generator current (transformed at the cavity gap) required to achieve the demanded cavity voltage without beam when the cavity is on tune [8]. Since it depends on $Q_{L}$ (proportional) and the cavity voltage (inversely proportional), its value will be different for operation at $450 \mathrm{GeV}$ and $7 \mathrm{TeV}$ and will vary based on the rf voltage chosen in normal operation in the HiLumi LHC era. Its maximum value of 7.2 is reached with operation settings of $Q_{L}=60000$ and $V_{c}=1.5 \mathrm{MV}$ at $7 \mathrm{TeV}$. Its lowest value of 3.6 results from operation with $Q_{L}=20000$ and $V_{c}=1 \mathrm{MV}$ at $450 \mathrm{GeV}$.

[8] F. Pedersen, Beam loading effects in the CERN PS booster, IEEE Trans. Nucl. Sci. 22, 1906 (1975).

[9] HiLumi LHC beam is defined as $1 \mathrm{~ns} 4$-sigma bunch length (corresponding to a $1.12 \mathrm{~ns}$ full base width for a parabolic line density distribution), $25 \mathrm{~ns}$ bunch spacing, $2.2 \times 10^{11}$ protons per bunch, and 2808 bunches per beam, amounting to $1.11 \mathrm{~A} \mathrm{DC}$, thus resulting in an rf component of beam current of $1.81 \mathrm{~A}$, assuming a parabolic line density. 
[10] D. Boussard, Control of cavities with high beam loading, IEEE Trans. Nucl. Sci. 32, 1852 (1985).

[11] D. Boussard and T.P.R. Linnecar, CERN Report No. CERN-LHC-Project-Report-316, 1999.

[12] P. Baudrenghien, G. Hagmann, J. Molendijk, R. Olsen, A. Rohlev, V. Rossi, D. Stellfeld, D. Valuch, and U. Wehrle, CERN Report No. LHC-PROJECT-Report-906, 2006, https://cds.cern.ch/record/971742.

[13] P. Baudrenghien, T. Mastoridis, and J. Molendijk, CERN Technical Report No. CERN-ATS-Note-2012-025 PERF, 2012.

[14] D. Boussard and G. Lambert, Reduction of the apparent impedance of wide band accelerating cavities by rf feedback, IEEE Trans. Nucl. Sci. 30, 2239 (1983).

[15] F. Pedersen, CERN Report No. CERN-PS-92-59-RF, 1992, https://cds.cern.ch/record/244817/export/hx?ln=en.

[16] At injection, a low rf voltage is required (0.75 MV or $1 \mathrm{MV}$ per cavity) but it is necessary to react quickly to injection phase and energy errors. A low $Q_{L}$ is therefore preferred. In physics, there is no need to modulate the rf but a large voltage is required (2 MV per cavity). A large $Q_{L}$ (60000) is therefore favorable. The main couplers move from low to high $Q_{L}$ position once the filling is complete, and before the start of the acceleration ramp.

[17] T. Mastorides, C. Rivetta, J. D. Fox, D. V. Winkle, P. Baudrenghien, and J. Tuckmantel, in Proceedings of the 11th European Particle Accelerator Conference, Genoa, Italy (CERN, 2008) (CERN Report No. SLAC-PUB-13291, 2008).

[18] D. Van Winkle, J. Fox, T. Mastoridis, C. Rivetta, P. Baudrenghien, A. Butterworth, and J. Molendijk, Feedback configuration tools for LHC low level rf system, in Proceedings of the 23rd Particle Accelerator Conference, Vancouver, Canada, 2009 (IEEE, Piscataway, NJ, 2009).

[19] D. Van Winkle, J. Fox, T. Mastorides, C. Rivetta, P. Baudrenghien, A. Butterworth, and J. Molendijk, in
Proceedings of the 1st International Particle Accelerator Conference, Kyoto, Japan (CERN, 2010).

[20] In accelerator physics, the effective impedance refers to the overlap between the object impedance and the bunch mode spectrum. In controls theory, it refers to the cavity impedance reduced by the rf feedback. In this work the effective impedance of the cavity follows the accelerator physics definition, but with the cavity impedance replaced by its value reduced by the feedback.

[21] A. Chao, Physics of Collective Beam Instabilities in High Energy Accelerators (John Wiley \& Sons, Inc., New York, 1993).

[22] F. J. Sacherer, A longitudinal stability criterion for bunched beams, IEEE Trans. Nucl. Sci. 20, 825 (1973).

[23] K. Robinson, Report No. CEAL-1010, 1964.

[24] P. Baudrenghien and T. Mastoridis, CERN Report No. CERN-ATS-2012-147, https://cds.cern.ch/record/ 1460930.

[25] P. Baudrenghien, CERN Report No. CERN-AB-Note2007-011, 2007.

[26] P. Baudrenghien, A. C. Butterworth, M. Jaussi, T. Mastoridis, G. Papotti, E. N. Shaposhnikova, and J. Tuckmantel, Longitudinal emittance blow-up in the LHC, in Proceedings of the 2nd International Particle Accelerator Conference, San Sebastiáán, Spain (EPS-AG, Spain, 2011).

[27] P. Baudrenghien and T. Mastoridis, Longitudinal emittance blowup in the large hadron collider, Nucl. Instrum. Methods Phys. Res., Sect. A 726, 181 (2013).

[28] J. F. Esteban Muller, P. Baudrenghien, A. Lasheen, T. Roggen, E. Shaposhnikova, and H. Timko, Report No. CERN-ACC-NOTE-2016-0001, 2016.

[29] H. Timko, Operational and beam dynamics aspects of the rf system in 2015, in 5th Evian Workshop, CERN-ACC-2015376, CERN, Geneva, Switzerland, https://cds.cern.ch/ record/2156966? $\ln =$ en. 